\title{
Mini-Bentall Surgery: The Right Thoracotomy Approach
}

\author{
Manish Jawarkar, M.Ch., Pratik Manek, M.Ch., Vivek Wadhawa, M.Ch., Chirag Doshi, M.Ch. \\ Department of Cardio Vascular and Thoracic Surgery, U. N. Mehta Institute of Cardiology and Research Center, B. J. Medical College, Ahmedabad, India
}

\section{ARTICLE INFO}

Received April 27, 2021

Revised July 5, 2021

Accepted August 5, 2021

Corresponding author

Manish Jawarkar

Tel 91-079-22684200

Fax 91-079-22682092

E-mail manishjawarkar@gmail.com

ORCID

https://orcid.org/0000-0003-3721-3547

\begin{abstract}
Surgeons are increasingly using the right mini-thoracotomy approach to perform aortic valve surgery. This approach has shown better results in terms of blood loss and length of hospital stay than the sternotomy approach. For selected patients requiring aortic root and ascending aorta surgery, a right mini-thoracotomy approach may prove beneficial. In our technique, we placed a 5-cm horizontal skin incision in the right second intercostal space. Femoro-femoral cardiopulmonary bypass was established. A valved aortic conduit was used for aortic root replacement. The patient's postoperative course was uneventful, with a short hospital stay. This technique offers a minimally invasive approach to aortic root and ascending aorta surgery with easy adaptability and reduced costs.
\end{abstract}

Keywords: Right mini-thoracotomy, Aortic root replacement, Aortic valve, Surgery

\section{Introduction}

The Bentall procedure, which was originally described by Bentall and De Bono [1] in 1968, is usually done through median sternotomy. Aortic valve replacement (AVR) via a right mini-thoracotomy has been shown to decrease blood loss and overall hospital length of stay as compared to AVR via sternotomy [2]. However, there is a dearth of substantial evidence regarding the use of mini-thoracotomy for replacement of the aortic root and ascending aorta. In this article, we describe our technique of performing elective surgery using the modified Bentall procedure through a right mini-thoracotomy in selected patients requiring aortic root and ascending aorta replacement.

\section{Preoperative data}

A 55-year-old male patient, with a known history of hypertension and chronic smoking, presented with dyspnea on exertion (New York Heart Association class II). The patient's preoperative work-up included routine blood investigations, chest radiography, electrocardiography, echocardiography, and computed tomography (CT) aortography. The preoperative echocardiogram was suggestive of left ventricular (LV) dilatation with mild LV dysfunction, severe aortic regurgitation, and aortic root dilatation (aortic root, $70 \mathrm{~mm}$; sinotubular junction, $66 \mathrm{~mm}$ ). A preoperative $\mathrm{CT}$ aortogram is useful to determine the extent of surgery required based on the dimensions, to evaluate any peripheral vascular disease, and to assess the position of the ascending aorta relative to the right border of the sternum, the distance of the aorta from the sternum, and the alpha angle (i.e., the angle of the inclination of ascending aorta with the midline). The favorable subset of patients comprises those with $\geq 50 \%$ of the aorta rightward of the right sternal border, a distance of the ascending aorta from the sternum of $\leq 10 \mathrm{~cm}$, and an alpha angle of $\geq 45^{\circ}$. The findings of the preoperative CT aortogram were as follows: aortic annulus, $31.5 \times 23 \mathrm{~mm}$; aortic root, $56.4 \times 49.2 \times 47.2 \mathrm{~mm}$; ascending aorta, $45.3 \times 41.4 \mathrm{~mm}$; arch of the aorta, $29.6 \times 27.3 \mathrm{~mm}$. Relative contraindications at U. N. Mehta Institute of Cardiology and Research Centre include patients with small aortic roots, aortic dissection or intra-mural hematoma, and surgical procedures requiring aortic valve preservation.

\section{Technique}

\section{Positioning and exposure}

The patient is placed in the supine position on the operating table with a left lateral tilt. External defibrillator pads are placed posteriorly and on the left lateral chest wall. An 
approximately 5- to 7-cm skin incision is placed horizontally along the right second or third intercostal space anteriorly (Fig. 1A). The selection of the intercostal space depends on the location of aortic annulus relative to the mediastinum. If the annulus is too low and pushed downwards, we open the third intercostal space. The right pleura is entered and a chest retractor is placed. A rib spreader is used for optimal exposure. However, if necessary, costal cartilage disarticulation can be done. If the thymus is thick and large, then it is excised or pushed posteriorly. The pericardium over the aorta is opened vertically and the incision is extended in a curvilinear fashion cranially up to the pericardial reflection over the aorta and caudally towards the right ventricular outflow tract. As many pericardial stays as required are placed for better exposure of the operative field, which should be hitched tightly with skin drapes.

\section{Femoral cannulation}

The femoral vessels are then exposed using a 2-cm transverse incision in the groin. An appropriate-sized arterial cannula is placed in the common femoral artery. An appropriate-sized venous cannula is placed into the femoral vein via the Seldinger technique, and the cannula advanced to the superior vena cava under transesophageal echocardiography (TEE) guidance (Fig. 1B). Cardiopulmonary bypass $(\mathrm{CPB})$ is established.

\section{Cardioplegia and aortic root dissection}

An aortic cross-clamp (conventional aortic cross-clamp or Cygnet [Vitalitec, Balgheim, Germany]) is applied through the same incision proximal to the origin of the innominate artery on transient low CPB flow, and antegrade modified cold blood cardioplegia is delivered directly to the coronary ostia. LV venting is done via the right superior pulmonary vein. The aneurysmal ascending aorta is resected and coronary buttons are harvested (Fig. 2A). The aortic valve leaflets are excised and the annulus is sized.

\section{Placement of the valved conduit}

Annular suturing is done using Ethibond 2-0 pledgeted sutures with the pledget on the aortic side. An appropriate-sized valved aortic conduit is placed.

\section{Coronary reimplantation and distal anastomosis}

Once the conduit is secured to the annulus, the left coronary button is reimplanted in the appropriate position using running 6-0 polypropylene sutures (Fig. 2B). Distal anastomosis with the ascending aorta is performed with 4-0 polypropylene continuous double-layer sutures. After the conduit is secured in its position, the right coronary button is reimplanted (Fig. 3A). For the entire anastomosis, long monoshaft instruments are recommended, and if
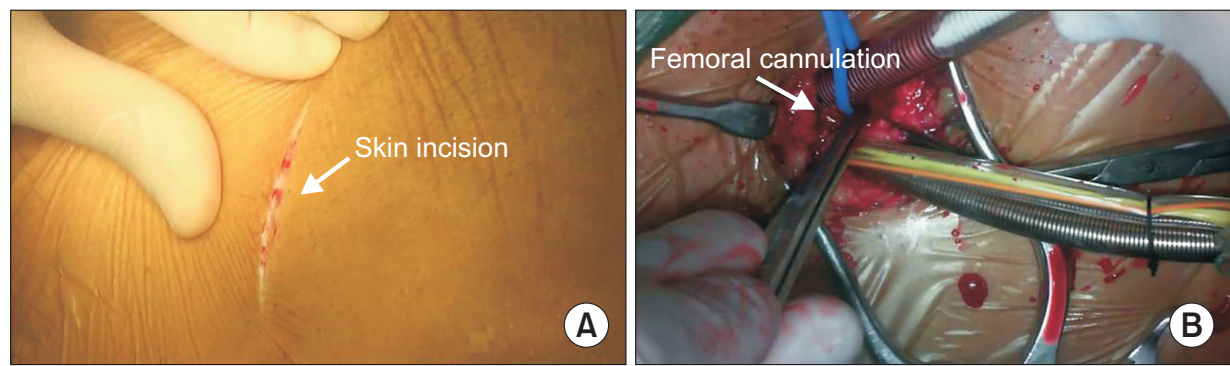

Fig. 1. (A) Skin incision. (B) Formal cannulation. Written informed consent for publication of this image was obtained from the patient.
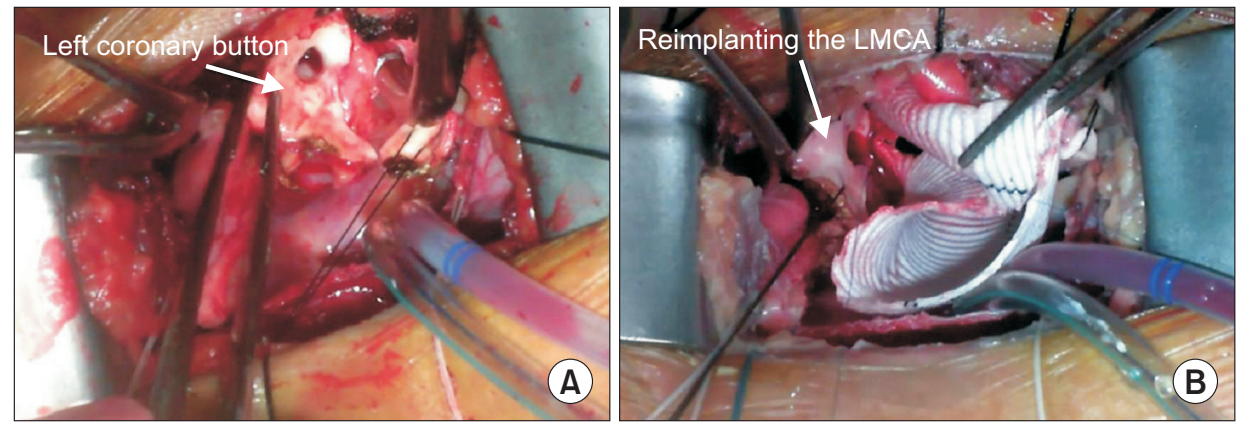

Fig. 2. (A) Left coronary button. (B) Reimplanting the left main coronary artery (LMCA). Written informed consent for publication of this image was obtained from the patient. 

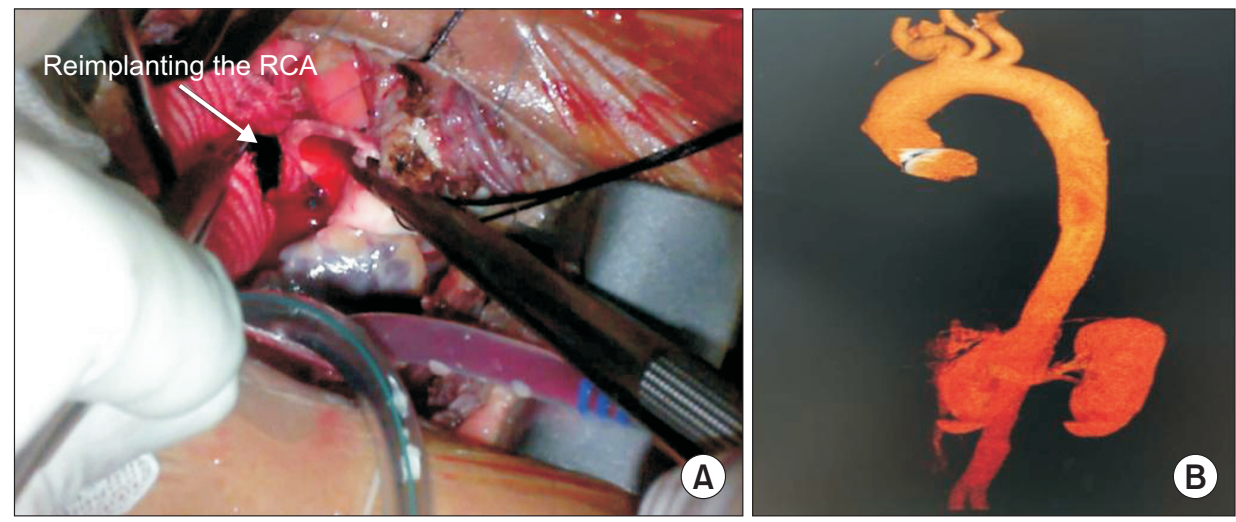

Fig. 3. (A) Reimplanting the right coronary artery (RCA). (B) Aortogram. Written informed consent for publication of this image was obtained from the patient.

needed, a knot pusher should be used. All the suture lines are checked and tightened if they are found to be loose during aortic cross-clamping, and surgical glue is used along all the suture lines before releasing the aortic crossclamp. When the tissue is friable and fragile, we perform the posterior suture line of the coronary button in 2 layers. The aortic cross-clamp time was 96 minutes and the $\mathrm{CPB}$ time was 112 minutes.

\section{Weaning off cardiopulmonary bypass}

A pacing wire is placed, de-airing is done, and the aortic cross-clamp is removed. De-airing should be done under TEE guidance by placing a root cannula in the graft conduit secured by a pledgeted prolene suture. The patient is gradually weaned off bypass. The femoral cannulae are removed and the femoral vessels are repaired under visualization. After adequate hemostasis, a chest tube is placed through a stab incision in the mid-axillary line and temporary pacing wires are brought out. The wound is closed in layers.

\section{Postoperative course}

The patient was extubated in the postoperative intensive care unit (ICU) after 6 hours. The patient ambulated in the ICU on day 1 , the chest drain was removed, 2-dimensional echocardiography was done, and patient was transferred to the postoperative ward. A CT aortogram was done on the third postoperative day and revealed satisfactory repair (Fig. 3B). The patient was discharged on postoperative day 4 on oral anticoagulants. The patient is receiving routine follow-up, is asymptomatic, and is carrying out his daily activities. The patients provided informed consent regarding the publication of the clinical findings and images.

\section{Discussion}

Minimally invasive AVR is a well-established surgical procedure and has several advantages over AVR through sternotomy in terms of decreased blood loss, length of hospital stay, early recovery of pulmonary function, and a rapid return to daily activities [3]. This has further expanded the scope of using right mini-thoracotomy for concomitant replacement of the ascending aorta. Lamelas and LaPietra [4] described the technical details of right mini-thoracotomy AVR with ascending aortic and proximal arch aneurysm repair. In their series, they observed a shorter median hospital stay. However, there is scarce evidence regarding the use of right mini-thoracotomy for Bentall surgery. Johnson et al. [5] in a retrospective review of 7 patients, described elective Bentall procedures performed through right mini-thoracotomy. In that series, all patients underwent surgery using video guidance, and automated suturing technology was used in some cases. Total circulatory arrest (TCA) was used in all cases of distal anastomosis.

In the case described herein, we used conventional instruments and suturing techniques. Neither rib disarticulation was done, nor was the right internal mammary artery (RIMA) sacrificed. However, if exposure is suboptimal, the costal cartilage can be disarticulated or the RIMA can be sacrificed. We did not use TCA for distal anastomosis. In our technique, we used a conventional aortic crossclamp placed through the same incision. However, this technique may reduce the exposure of the working field for the surgeon; therefore, if needed, a flexible aortic crossclamp (signet ring clamp) or Chitwood clamp can also be placed through the same incision or through a separate incision on the mid-axillary line. This technique is helpful if the diseased area is limited to the upper ascending aorta. If the need arises to perform surgery on the hemiarch, the same approach can be used, but the patients must be 
placed on TCA. The same composite valve-graft prosthesis used in open modified Bentall surgery was used. The CPB time in our patient was 112 minutes, which is much shorter than the mean CPB time of 202.9 \pm 47.8 minutes in the case series by Johnson et al. [5]. Similarly, the cross-clamp time in our case was 96 minutes, which is meaningfully shorter than the mean cross-clamp time of $161.9 \pm 32.1$ minutes reported in their study. The reduction in the aortic crossclamp time and CPB time may be due to the increasing experience with this technique and standardization of the operative steps. The length of the hospital stay and time to extubation in our case were comparable. The patient had an uneventful postoperative course and was discharged on the fourth postoperative day. The shortened ICU and hospital stay helped to reduce the overall costs when compared to conventional sternotomy. Better cosmesis also adds to patients' satisfaction. When selecting this approach, we avoid patients who require aortic arch repair, concomitant coronary artery bypass grafting, or other valvular surgery. It is also preferable not to use this technique to operate in patients with peripheral vascular disease.

\section{Conclusion}

Aortic root replacement can be performed concomitantly with replacement of the ascending aorta and proximal arch through right mini-thoracotomy in carefully selected patients. This technique can be easily adopted and reproduced, and it is cost-effective.

\section{Conflict of interest}

No potential conflict of interest relevant to this article was reported.

\section{ORCID}

Manish Jawarkar: https://orcid.org/0000-0003-3721-3547

Pratik Manek: https://orcid.org/0000-0001-5884-8002

Vivek Wadhawa: https://orcid.org/0000-0003-0069-2644

Chirag Doshi: https://orcid.org/0000-0002-9526-5217

\section{References}

1. Bentall H, De Bono A. A technique for complete replacement of the ascending aorta. Thorax 1968;23:338-9.

2. Perrotta S, Lentini S, Rinaldi M, et al. Treatment of ascending aorta disease with Bentall-De Bono operation using a mini-invasive approach. J Cardiovasc Med (Hagerstown) 2008;9:1016-22.

3. Bowdish ME, Hui DS, Cleveland JD, et al. A comparison of aortic valve replacement via an anterior right minithoracotomy with standard sternotomy: a propensity score analysis of 492 patients. Eur J Cardiothorac Surg 2016;49:456-63.

4. Lamelas J, LaPietra A. Right minithoracotomy approach for replacement of the ascending aorta, hemiarch, and aortic valve. Innovations (Phila) 2016;11:301-4.

5. Johnson CA Jr, Siordia JA, Wood KL, Robinson DA, Knight PA. Right mini-thoracotomy Bentall procedure. Innovations (Phila) 2018;13: 328-31. 